\title{
Protocol
}

\section{Development and evaluation of a culturally tailored education module for type 1 diabetes in a resource-constrained setting: protocol for a mixed-methods study}

\author{
Latika Rohilla1, Devi Dayal ${ }^{1 *}$, Prahbhjot Malhi' ${ }^{1}$, \\ Bhavneet Bharti' ${ }^{1}$, Sukhpal Kaur ${ }^{2}$, Manju Dhandapani
}

${ }^{1}$ Department of Pediatrics, Postgraduate Institute of Medical Education and Research, Chandigarh, India
${ }^{2}$ National Institute of Nursing Education, Postgraduate Institute of Medical Education and Research, Chandigarh, India

Received: 26 June 2021

Revised: 16 July 2021

Accepted: 19 July 2021

\author{
*Correspondence: \\ Dr. Devi Dayal, \\ E-mail: drdevidayal@gmail.com
}

Copyright: (c) the author(s), publisher and licensee Medip Academy. This is an open-access article distributed under the terms of the Creative Commons Attribution Non-Commercial License, which permits unrestricted non-commercial use, distribution, and reproduction in any medium, provided the original work is properly cited.

\begin{abstract}
Background: Diabetes education is the key to successful diabetes management. There is a need for an education module for type 1 diabetes (T1D) that is culture-specific and suited to resource constraints.

Methods: A mixed-methods study will be conducted, in three phases, to develop and evaluate a culturally tailored diabetes education module for Indian children with T1D and their families. During the first phase, a qualitative study among health professionals and families of children with T1D for need assessment will be conducted. During the second phase, based on the themes from the last phase, an educational module will be developed. The third phase will involve an evaluation of the content, feasibility and effectiveness of the proposed module. The content evaluation will be done using the standardized 'suitability assessment of materials' checklist. For feasibility, a mixed-method approach will be used with iterative cycles of satisfaction scale, semi-structured interview and feasibility and observation checklist. The module will be revised after each cycle till no new changes are suggested. The effectiveness will be assessed by a quasiexperimental controlled trial assessing glycemic control, health-related quality of life, clinically important events and self-management practices in T1D children at baseline and three and six months.

Conclusions: This study aims at development and validation of a novel culturally tailored diabetes education module for children with T1D, suited to their resource constraints. A module designed with the inputs from all stakeholders, and evaluated using iterative cycles, has the potential to suit the dynamic nature of diabetes management in children. Trial registration: Trial registration number is CTRI/2021/04/032739.
\end{abstract}

Keywords: Type 1 diabetes, Glycemic control, Health education, Needs assessment, Self-management, Children

\section{INTRODUCTION}

Diabetes education is the master key to successful diabetes management. ${ }^{1}$ A quality assured structured education program is acknowledged as a right of all children with diabetes. ${ }^{2}$ Diabetes education has the potential to improve glycemic control as well as health-related quality of life
(HRQOL), especially when it is continuous and is embedded in the management protocol since diagnosis with reinforcements at frequent intervals. ${ }^{3}$

Diabetes calls for a multitude of tasks for self-management according to the changes in diet, activity and physiology of children. ${ }^{4}$ The challenge faced by the developing 
countries is even more. Families have a tough time meeting the financial burden of the basic tools like glucose strips, needles and insulin injections, involved in the daily treatment tasks. ${ }^{5}$ Also, negligible support is offered to these children by the government even in big countries like India. ${ }^{6}$

Cultural and language differences can often hinder diabetes education. It is important to meet the local practices and ensure that the diabetes education plan suits the literacy levels of different people. So, there is a felt need for a customized and adaptable education module according to age, stage of disease, maturity and understanding level, culture and learning pace. ${ }^{1}$ Till date, a standard low literacy educational module is missing to cater to the individual needs of the parents of the children with T1D. ${ }^{7,8}$ The one size fits all approach in diabetes education often fails to help and more culturally appropriate educational interventions are the need of the hour. ${ }^{9,10}$

\section{Problems specific to India}

In a low-resource country like India, there are certain specific well-recognized factors such as the high cost of diabetes-related items, deficient medical insurance and social security and inadequate health care infrastructure causing difficult access to diabetes education. ${ }^{11}$

In addition to this, people with T1D face differential treatment and harassment at school, college and employment. Social discrimination is particularly pronounced against females with chronic illnesses. ${ }^{12}$ Cultural differences, health beliefs and limited educational background have been identified as major barriers to adequate diabetes care in a family with T1D. ${ }^{13}$ Cultural factors specific to our part of the world that can lead to poor glycemic control in children are as follows: ${ }^{14,15}$

Role of grandparents in child upbringing: educating the parents for diabetes management fails to bring desired results in extended families, where parents have a lesser say in deciding treatment and no single person is responsible for the care of the child; a patriarchal society with a lesser decision-making in the hands of the mother of the child; constant desire to get rid of insulin injections; a strong affinity for traditional remedies leads patients to waste time and resources and lack of focus on insulin regimens; dietary taboos mark particular foods as hot or cold and are consequently consumed according to seasons, e.g. eggs are not eaten during summers as they are considered hot food, such taboos reduce the food options that a diabetic child has around the year; religion specific norms which indulge in feasting during some festivals and fasting during others; inadequate knowledge and confidence leading them to seek information from incompetent sources such as neighbors and friends; and inappropriate information on disease cure and remedies, easily available on social media and other online platforms, which is poorly supported by evidences and societal acceptance for a chronic disease involving use of needles. ${ }^{14}$ Parents and children avoid disclosing their illness to anyone at school or in society. This may lead to an inability to receive others' help when required, especially during severe hypoglycemic events. ${ }^{14,15}$

Although there are many validated educational programs for adults with type 2 diabetes (T2D), very few are validated for T1D. These are the diabetes teaching and treatment programme (DTTP)-Germany, dose adjustment for normal eating (DAFNE)-UK and the diabetes literacy and numeracy education toolkit (DLNET)-USA. In the developing world, only Malaysia, Korea and Iran have culturally tailored education modules developed in the local language for T2D, that too for adults with T2D. ${ }^{16,18}$

Thus, there is a felt need for a validated educational program that is tailored to overcome cultural, educational and financial barriers specific to low-resource settings like India for the optimal management of children with T1D.

\section{Objectives of this study}

\section{Primary}

The primary objectives of this study were to explore the parents' and health care professional's perspectives about the desirable and essential features of a diabetes education module; to develop a culturally tailored education module for children with T1D in a resource-constrained setting and to evaluate the content and feasibility of the developed module and to evaluate the effectiveness of the developed education module among parents of children with T1D in terms of glycemic control.

\section{Secondary}

The secondary objectives were to evaluate the effectiveness of the developed education module in terms of HRQOL of children, clinically important events and self-management practices related to diabetes care at home.

\section{METHODS}

This study aims to develop and evaluate a culturally tailored education module for T1D in a resourceconstrained setting. Figure 1 depicts the flow diagram of the study protocol. It has been designed according to the medical research council (MRC) framework for developing complex interventions. ${ }^{19}$

According to this framework, there are five steps of evaluating complex interventions like disease-specific health education. These are as follows:

Theory: Identifying existing theory and evidence to ensure selection of best components for the intervention. 
Modelling: Identifying key components of the intervention and exploring how it may work in practice.

Exploratory trial: Describing the components, testing its effects and continuing to revise the intervention.
Long term implementation: Bigger, multi-centric studies to replicate the results.

This study, as part of a PhD thesis, is designed to execute the first four steps of the above-mentioned framework.

Definitive randomised controlled trial (RCT): Testing the fully developed intervention in an appropriate controlled trial.

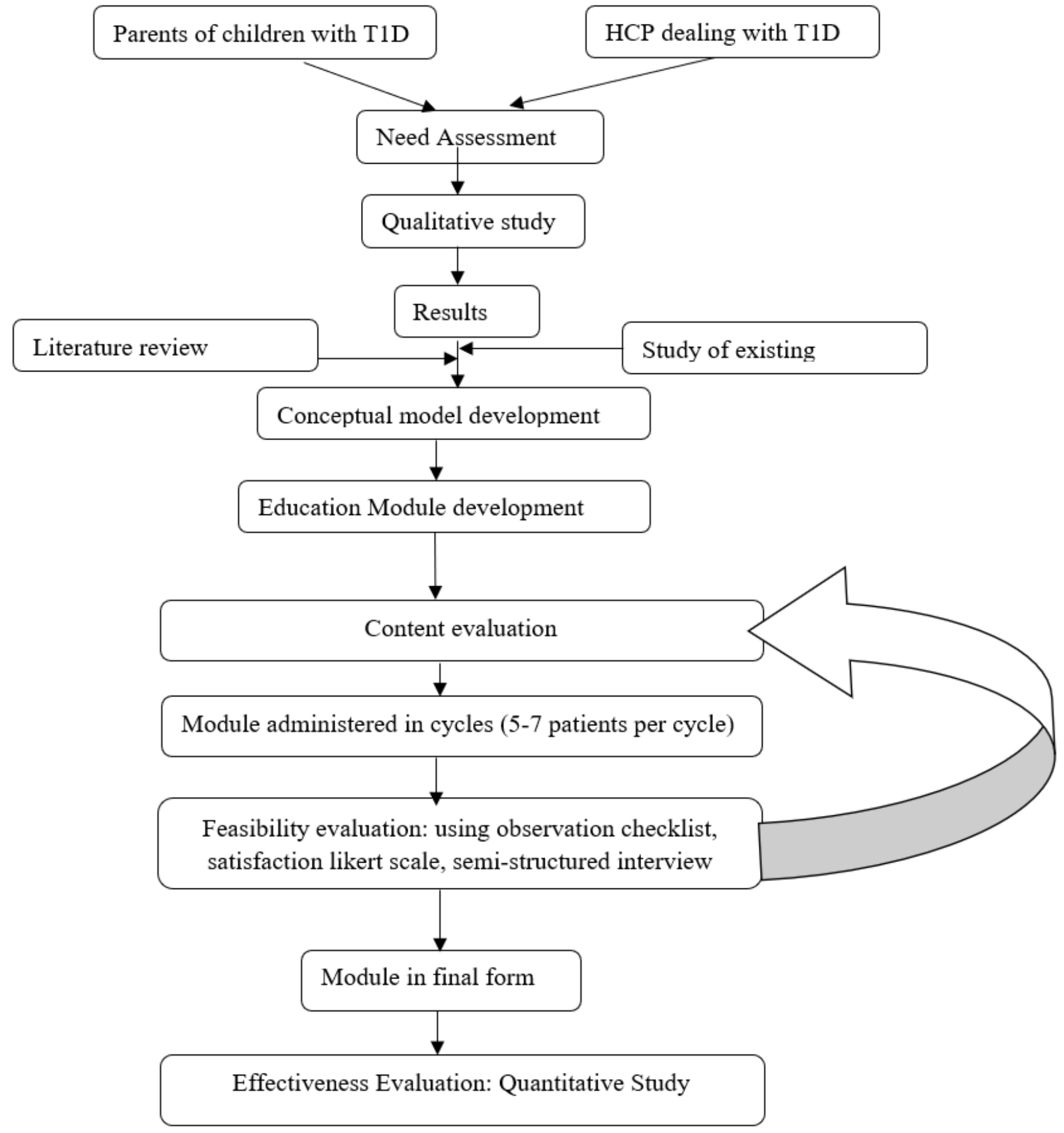

Figure 1: Flow diagram of the study protocol. 
This study will be a mixed-methods study using both qualitative and quantitative methods at different steps. The study duration will be four years. The study will be carried out in three phases. In the first phase, a qualitative study will be conducted for need assessment among the children with T1D and their families and the health care professionals dealing with them. During the second phase, the themes generated from the first phase, existing behavioural theories, and literature review will be used to develop a conceptual model for the effectiveness of diabetes education. This model will then be used to build up and propose a culturally tailored diabetes education module for children with $\mathrm{T} 1 \mathrm{D}$ in resource-constrained settings.

The third phase will include the evaluation of the proposed module in terms of its content and feasibility, and effectiveness. For content and feasibility evaluation, a predefined and structured approach will be followed by a multi-disciplinary team for the refinement of the module. Then, using a quantitative approach, the effectiveness of the education module in terms of glycemic control and other related variables will be tested using a quasiexperimental controlled study among children admitted with T1D.

\section{Phase 1: Qualitative study for need assessment}

This phase will explore the patient perspectives about the desirable and essential features of a diabetes education intervention to suit their resource constraints. Along with this, this will explore the health professional perspectives on the features of the intervention so as to increase uptake and usage by such parents as well as the deliverability by the professionals. The duration of this phase shall be six months.

\section{Participants}

Parents, children with T1D and healthcare professionals (HCP) involved in the management of T1D children (nurses, doctors, dieticians, representatives from the nongovernmental organisations (NGOs) working in the region and primary healthcare workers) will be included in this phase.

\section{Inclusion criteria}

Parents and their children undergoing treatment for T1D, either on an in-patient or out-patient basis, will be included if the child's age is less than 13 years and they can speak Hindi, Punjabi or the English language. Healthcare and other professionals will be included if they are directly involved in the management of children with T1D. Children with associated diseases that may affect their quality of life such as cancer, mental retardation or developmental disorders will be excluded from the study.

\section{Data collection}

A qualitative study will be conducted. It will have focus group discussion (FGD) among members of the following sub-groups: families will be divided into the low, middle or upper class as per their socio-economic status according to the modified B. G. Prasad scale; diagnosis of T1D of children (old/new cases); clinicians from the departments of endocrinology and pediatrics of the institute; nurses and dieticians; representatives from NGOs working for diabetes in children; workers from primary health centers and school teachers where children with T1D are studying. ${ }^{20-22}$ Purposive sampling will be done to ensure maximum representation of different sub-groups. All participants will be invited for the FGDs only after obtaining written informed consent and assent will be taken from all children above eight years of age.

In-depth interviews will be planned for further exploration for those found unable to express themselves adequately during the FGDs. ${ }^{20}$ Time and venue of the FGDs will be selected as per participants' convenience. Each FGD will have five to seven participants. The data collection shall continue till data saturation is reached.

\section{Phase 2: Designing a structured educational module}

The themes generated from phase one of the study will be used to develop a conceptual model. The development of the structured educational module will be done based on the conceptual model, keeping in mind the general principles of education. This means that the module will be focused on enhancing all three components of learning, knowledge, attitude and skill. An extensive review of the scientific literature, available diabetes management guideline resources (e.g., American diabetes association, international society for pediatric and adolescent diabetes, international diabetes federation) and inputs from nurses, physicians and dietitians will be utilized to guide the content. The module shall target grade five reading level. The main aim during the development of the module will be to transform established concepts of diabetes education (insulin titration, carbohydrate counting, emergency management of hypoglycemia/hyperglycemia, sick day rules for children with T1D) so as to make them more culturally specific. ${ }^{1,2}$ For example, insulin titration according to carbohydrate intake can be explained by using currency concepts and comparing them to the Barter system of ancient India (a transaction in which, instead of money, goods or services are directly exchanged for other goods or services). The duration of this phase will be six months.

\section{Phase 3: Evaluation of the education module}

This phase will be conducted in three years. It will be further divided into three steps: content evaluation (six months), feasibility evaluation (six months) and effectiveness evaluation (two years) of the developed educational module. 


\section{Content evaluation}

The content evaluation will be done by a multi-disciplinary team of medical and nursing experts. This will be done using the suitability assessment of materials (SAM) which is a standardized tool for the assessment of the suitability of educational materials for low literacy skills. ${ }^{23,24}$ SAM has been validated by experts from different cultural backgrounds and is suitable for printed materials, illustrations, video and audio-taped instructions. It is a structured scoring sheet with a total of 22 factors under six domains, content, literacy demand, graphics, layout and typography, learning stimulation and motivation, and cultural appropriateness. Percentage scores are given to each factor, and it takes 30-45 minutes for evaluation of educational material using SAM that can categorize the content as superior, adequate, or not suitable. Content of the education module will be finalized after reaching a consensus among the experts according to the SAM scores.

\section{Feasibility evaluation: mixed-methods study}

A mixed-methods feasibility testing approach will be used with iterative cycles of testing the developed intervention. ${ }^{25,26}$ A satisfaction scale, semi-structured interview, feasibility and observation checklist will be used to assess the feasibility and usability of the education module in real-world settings.

\section{Participants}

The parents and their children with T1D will be included in this study. The purposive sampling technique will be used, and inclusion criteria will remain as in the previous phase.

\section{Data collection}

The developed education module will be given a trial run among families of children with T1D. Observations made by the investigators about the feasibility and inputs from the participants will be used to refine the module before the next cycle of trial. At least two iterative cycles with approximately 5-7 participants per cycle will be conducted. Figure 2 depicts the flow diagram of the feasibility evaluation. This process will continue till no new amendment is suggested by the audience. The following instruments will be used:

The satisfaction scale will be used to measure participant satisfaction. It will be a Likert scale developed according to the content of the module. The reliability and validity of the tool will be established as a part of this study. It will be first pilot-tested to garner feedback related to clarity, relevance and completion time. The questionnaire will be administered to the participants after the module completion.

The semi-structured interview schedule will be used immediately following module completion, participants will be asked a set of open-ended questions related to the content and comprehensibility of the module. This will be developed based on the tool developed in a study by Connan et al. ${ }^{25}$ It will include questions on what the participants found easy or difficult to understand; liked or disliked about the module; found most or least useful; found easy or hard to use and would like to change or add. Participants will be asked about any technical issues they encountered. Open-ended questions for feedback will be used to elicit more information.

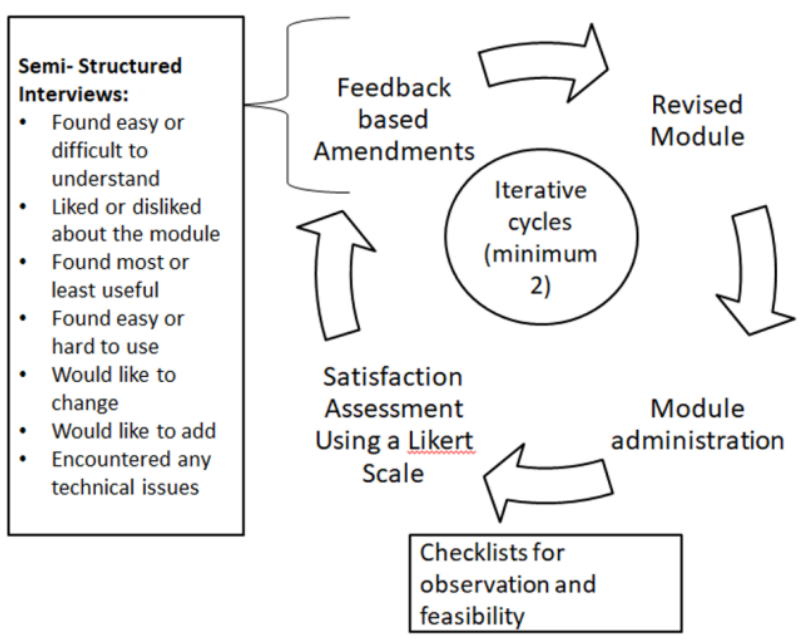

Figure 2: Flow diagram of feasibility evaluation.

The feasibility and observation checklist will be developed according to the feasibility framework to suit the developed education module. ${ }^{26}$ The reliability and validity of this tool will be established as a part of this study. The feasibility and the observation checklist will be completed during the administration of the education module. This tool will have six areas of focus, acceptability, demand, implementation, practicality, integration and limited efficacy.

\section{Effectiveness evaluation: quantitative study}

A quasi-experimental controlled trial will be conducted to assess the effectiveness of the education module on the glycemic control of children with T1D. Figure 3 describes the flow diagram of the effectiveness evaluation study. The null hypothesis is education module will have no significant effect on the glycemic control of the children with T1D at 0.05 level of significance.

The secondary outcome variables that will be assessed are HRQOL of children, clinically important events and selfmanagement practices related to diabetes care at home.

\section{Participants}

Parents and children with T1D will be selected as per the same inclusion criteria as explained in the first phase of the study. As per the relevant literature review, this is the first planned control trial to evaluate the effectiveness of a diabetes education module in T1D in India as well as the 
South-East Asia region. Hence, the sample size was calculated on the basis of a recent study on patientcentered education among adolescents with T1D done in Sweden. ${ }^{27}$ Assuming that the education module will lead to a difference in the HbA1c of $0.8 \%$, with the power of the study was 0.80 , level of significance $p=0.05$, confidence interval of $95 \%$, the sample size calculated to be 60 (30 in each arm). Expecting a loss to follow up rate of $20 \%$ and applying a correction factor for the same, the required sample size will be 72 with 36 in each group. Keeping in mind the current pattern of indoor admissions, eight to ten patients will be admitted every month. So, keeping a fair extra margin, the total enumeration sampling technique will be used for six months for each group.

\section{Routine Diabetes Education for the control group}

The control group will be administered diabetes education as per the current prevalent practice of the department. The parents of the admitted children are offered lecture group sessions of one-hour duration, every day till discharge using power point presentations in English and Hindi. Along with this, they are given a printed booklet (available in English and Hindi). The sessions as well as the booklet include diabetes education on the following aspects:

Basics of diabetes: Explaining T1D, its pathophysiology, symptoms and complications forms the basics of diabetes.

Home management of children with T1D: Types of insulin, management of hypoglycemia, hyperglycemia, sick day rules for glycemic control, insulin titration and basic carbohydrate counting (giving examples of foods with one carbohydrate exchange unit, 15 grams carbohydrates).

Practical skills related to diabetes: Usage, handling and storage of glucometer, insulin pens/syringes, follow up care are the practical skills related to diabetes.

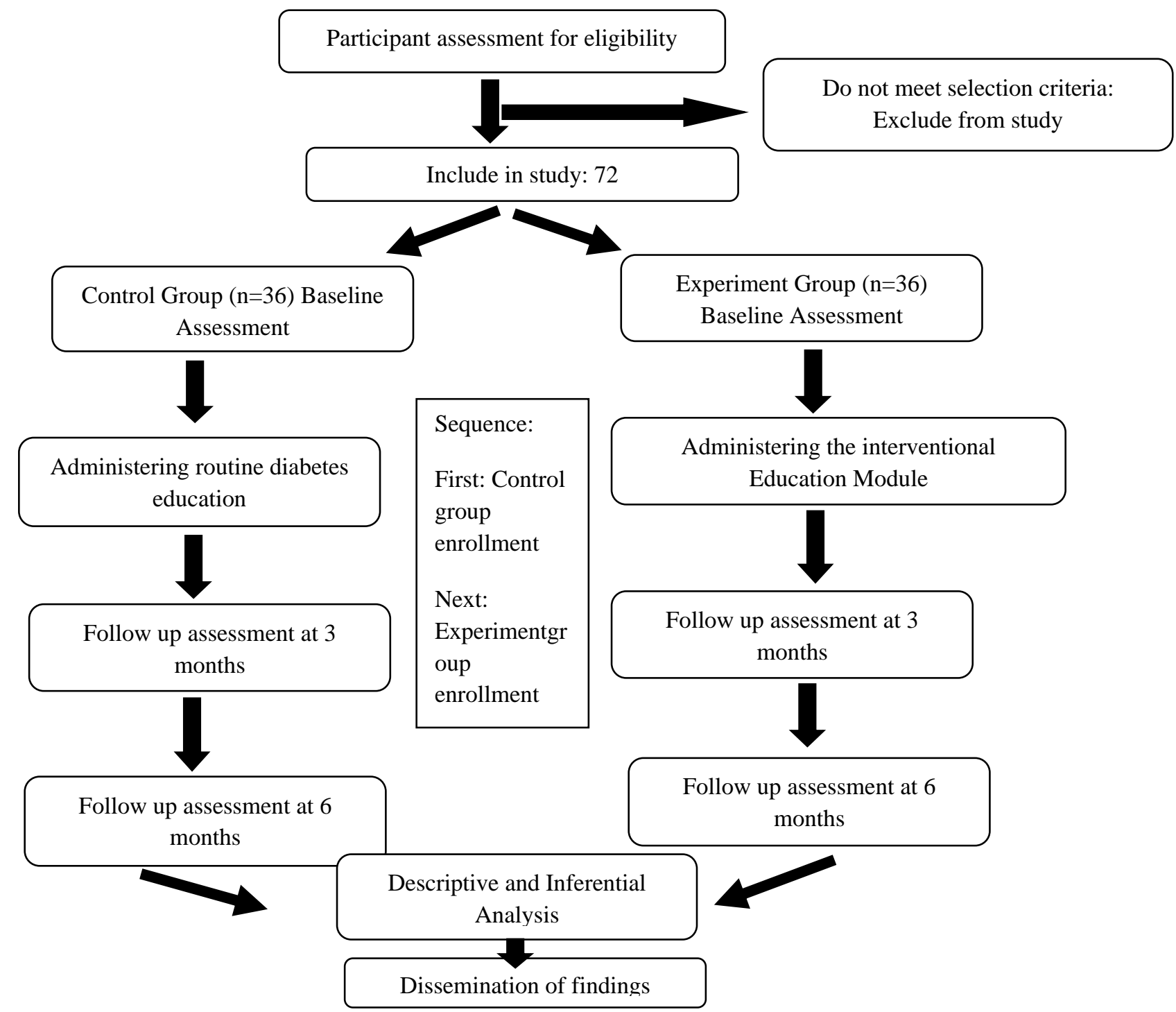

Figure 3: Flow diagram for effectiveness evaluation study. 
Table 1: Timeline of the study protocol.

\begin{tabular}{|c|c|c|c|c|c|c|c|c|c|}
\hline \multirow[b]{2}{*}{ Phase of the study } & \multirow[b]{2}{*}{ Task } & \multicolumn{2}{|c|}{ Year 1} & \multicolumn{2}{|c|}{ Year 2} & \multicolumn{2}{|c|}{ Year 3} & \multicolumn{2}{|c|}{ Year 4} \\
\hline & & $\begin{array}{l}\text { Jan- } \\
\text { June }\end{array}$ & $\begin{array}{l}\text { July- } \\
\text { Dec }\end{array}$ & $\begin{array}{l}\text { Jan- } \\
\text { June }\end{array}$ & $\begin{array}{l}\text { July- } \\
\text { Dec }\end{array}$ & $\begin{array}{l}\text { Jan- } \\
\text { June }\end{array}$ & $\begin{array}{l}\text { July- } \\
\text { Dec }\end{array}$ & $\begin{array}{l}\text { Jan- } \\
\text { June }\end{array}$ & $\begin{array}{l}\text { July- } \\
\text { Dec }\end{array}$ \\
\hline \multirow{2}{*}{$\begin{array}{l}\text { Evaluation of the } \\
\text { routine education } \\
\text { practices }\end{array}$} & $\begin{array}{l}\text { Control group } \\
\text { enrollment }\end{array}$ & & & & & & & & \\
\hline & $\begin{array}{l}\text { Follow up at } 3 \text { and } 6 \\
\text { months }\end{array}$ & & & & & & & & \\
\hline Need assessment & $\begin{array}{l}\text { Qualitative } \\
\text { study }\end{array}$ & & & & & & & & \\
\hline Module development & $\begin{array}{l}\text { Education module } \\
\text { development }\end{array}$ & & & & & & & & \\
\hline \multirow{4}{*}{$\begin{array}{l}\text { Evaluation of the } \\
\text { developed module }\end{array}$} & $\begin{array}{l}\text { Content } \\
\text { evaluation }\end{array}$ & & & & & & & & \\
\hline & $\begin{array}{l}\text { Feasibility } \\
\text { evaluation }\end{array}$ & & & & & & & & \\
\hline & $\begin{array}{l}\text { Intervention group } \\
\text { enrollment }\end{array}$ & & & & & & & & \\
\hline & $\begin{array}{l}\text { Follow up at } 3 \text { and } 6 \\
\text { months }\end{array}$ & & & & & & & & \\
\hline
\end{tabular}

\section{Data collection}

The admitted children with T1D will be identified. Their families will be invited to participate in the study. They will then be administered either the routine diabetes education or the developed culturally tailored education module. The data collection will be done at baseline and during follow up at three and six months. Epicollect 5 will be used for data collection. It is free to use software that can help create a customized database suitable to the research purpose. It allows for offline data entry and has cloud storage of data. ${ }^{28}$ The tools have been finalized after a pilot study on ten percent of the sample (eight children) population and are described below:

Socio-demographic proforma, which includes data related to the education and occupation of parents, housing and socio-economic status of the family; clinical profile proforma which includes questions on their present glycemic control in terms of $\mathrm{HbA1c}$, height, weight, frequency of hypoglycemic and hyperglycemic events in last seven days, episodes of diabetic ketoacidosis in last three months, episodes of missed or wrong dose of insulin, and total daily dose of bolus and basal insulin; PedsQLTM pediatric quality of life inventory version 3.2-diabetes module will be used for assessing the HRQOL among the children, it is a standardized tool available in Hindi and can measure diabetes-specific HRQOL from both child's and parents' perspectives, it has 33 items under five domains, namely diabetes symptoms, treatment one, treatment two, worry and communication; ${ }^{29}$ diabetes self-management profile-self report-Hindi will be used for assessing the selfefficacy of the parents, cross-cultural adaptability of the tool for Indian children with T1D has been established by Barola et al. ${ }^{30}$ This standardized tool assesses five areas of diabetes management, namely, exercise, hypoglycemia management, diet, blood glucose monitoring and insulin administration. The tool has 24 items and rates each item between 0-4, with higher scores indicating better treatment adherence.

\section{Plan for data analysis}

For the quantitative analysis, data will be analyzed using SPSS 23.0 (IBM, Armonk, New York). All data will be presented in terms of means, frequency, standard deviation and confidence interval. A two sided $\mathrm{p}$ value of 0.05 will be considered statistically significant. Intention-to-treat analysis will be done to compare outcomes at baseline, three and six months. According to the normality and type of data, appropriate statistical tests will be used to compare the variables between two groups and within the group from baseline to follow up at three and six months. For the qualitative data, conventional content analysis will be applied for data analysis and interpretation.

\section{Ethical considerations}

The ethical approval of the study has been taken from the Institute Ethics Committee, PGIMER, Chandigarh, India, which is an independent body (INT/IEC/2021/SPL-252 dated 13 February 2021). The study has been registered with the clinical trials registry of India (CTRI/2021/04/032739). Informed written consent will be taken from the parents of the enrolled children. This study will involve the children with T1D and their parents. There will be no interference in the routine treatment plan of the patients. There will be strict adherence to the principles of the declaration of Helsinki (2013, 7th edition, Fortaleza). All participants will be informed about their participation in the research, the objectives of the study and the duration of their involvement in advance. Full autonomy will be provided to the participants for withdrawing from the study at any time without any adverse effects on their subsequent care. Informed written consent will be taken from the parents of the enrolled children. Assent will be 
taken from all children above the age of eight years. Confidentiality and anonymity of the participants will be ensured while data collection and reporting the results of the study.

\section{DISCUSSION}

Although special diabetes education programs are developed and evaluated in some countries, the picture is not uniform across the globe. ${ }^{1}$ Despite a vast literature highlighting the importance of diabetes health education, only a small number of group education programs have been tested and they too don't provide uniform evidence in terms of glycemic control. ${ }^{27,31}$ Thus, this study aims at the development and validation of a novel culturally tailored diabetes education module for children with T1D and their families in India.

This study is modeled on the MRC framework for developing complex interventions, including its first four steps. ${ }^{19}$ Accordingly, the qualitative need assessment and iterative cycles of refining the intervention involving the target population can help in ensuring that the module will be developed as per their concerns and needs. However, the last step of the MRC framework, long term implementation, multi-centric trials for replication of intervention effects are not possible as part of this study due to the logistic and financial restrictions. This is an important implication for future studies.

The present study is being planned at a tertiary-level pediatric hospital. The admitted children with T1D are kept in the same ward and the open infrastructure allows them to freely interact with each other round the clock. As contamination between the trial arms is inevitable in such a setup, RCT is not feasible. In such a situation, as spatial separation of the trial arms is not feasible, separating the trial arms temporally can help to mitigate contamination. ${ }^{32}$ The control and the interventional group will thus be enrolled sequentially. The investigator, LR, will be solely responsible for the administration of the interventions to both groups. She is the practicing diabetes nurse educator in the unit for the past three years. Assuming that the module development will evolve her too, the control group enrollment will be completed first before she indulges herself in the qualitative need assessment and module development phase to reduce the investigator bias. Table 1 shows the proposed timeline of the study protocol.

Quality diabetes education is the right of every child with diabetes. ${ }^{2}$ Therefore, all families admitted during the data collection period will be offered diabetes education irrespective of their desire to participate or refrain from the study. The education being implemented at that time (routine diabetes education or the newly developed education module, in control and intervention group, respectively) will be offered to all the admitted children, but the follow-up will be done only amongst the ones meeting the inclusion criteria.

\section{CONCLUSION}

To conclude, it can be said that, whereas the financial constraints cannot be tackled immediately, modifying the existing educational programs to ensure better understanding and reduce the risk of mismanagement by empowering the unlettered parents can be a significant turning stone in the management of T1D in a multicultural indian society. Therefore, this study aims to establish a culturally tailored education program for children with diabetes.

\section{ACKNOWLEDGEMENTS}

The authors would like to thank the postgraduate institute of medical education and research, Chandigarh for supporting this research.

\section{Funding: No funding sources}

Conflict of interest: None declared

Ethical approval: The ethical approval of the study has been taken from the Institute Ethics Committee, PGIMER, Chandigarh, India

\section{REFERENCES}

1. Phelan H, Lange K, Cengiz E, Gallego P, Majaliwa E, Pelicand $\mathrm{J}$, et al. ISPAD clinical practice consensus guidelines 2018: diabetes education in children and adolescents. Pediatr Diabetes. 2018;19(27):75-83.

2. American Diabetes Association. Children and adolescents: standards of medical care in diabetes2018. Diabetes Care. 2018;41(1):126-36.

3. Döğer E, Bozbulut R, Acar ASS, Ercan Ş, Uğurlu AK, Akbaş ED, et al. Effect of telehealth system on glycemic control in children and adolescents with type 1 diabetes. J Clin Res Pediatr Endocrinol. 2019;11(1):70-5.

4. Kichler JC, Seid M, Crandell J, Maahs DM, Franziska K, Driscoll KA, et al. The Flexible Lifestyle Empowering Change (FLEX) Intervention for self-management in adolescents with Type 1 Diabetes: Trial design and baseline characteristics. Contemp Clin Trials. 2019;66:64-73.

5. Sundberg F, Barnard K, Cato A, deBeaufort C, DiMeglio LA, Dooley G, et al. Managing diabetes in preschool children. Pediatr Diabetes. 2017;18(7):499-517.

6. Virmani A. Type 1 diabetes in India: the numbers show the way ahead. Indian Pediatrics. 2019;56(3):189-90.

7. Grey M. Interventions for children with diabetes and their families. Annu Rev Nurs Res. 2000;18:149-70.

8. Hilliard ME, Tully C, Monaghan M, Wang J, Streisand R. Design and development of a steppedcare behavioral intervention to support parents of young children newly diagnosed with type 1 diabetes. Contemp Clin Trials. 2017;62:1-10. 
9. Chatterjee S, Davies MJ, Heller S, Speight J, Snoek FJ, Khunti K. Diabetes structured self-management education programmes: a narrative review and current innovations. Lancet Diabetes Endocrinol. 2018;6(2):130-42.

10. Teft G. Numeracy skills in people with diabetes. Diabet Prim Care. 2015;17(5):218-20.

11. Wangnoo SK, Maji D, Das AK, Rao P V, Moses A, Sethi B, et al. Barriers and solutions to diabetes management : an Indian perspective. Indian J Endocr Metab. 2013;17(4):594-601.

12. Bhatia V, Arya V, Dabadghao P, Balasubramanian $\mathrm{K}$, Sharma K, Verghese N, et al. Etiology and Outcome of Childhood and Adolescent Diabetes Mellitus in North India. J Pediatr Endocrinol Metab. 2004;17(7):993-9.

13. Povlsen L, Olsen B, Ladelund S. Educating families from ethnic minorities in type 1 diabetes-experiences from a Danish intervention study. Patient Educ Couns. 2005;59(2):164-70.

14. Kalra S, Sridhar GR, Balhara YPS, Sahay RK, Bantwal G, Baruah MP, et al. National recommendations: psychosocial management of diabetes in India. Indian $\mathbf{J}$ Endocrinol Metab. 2020;17(3):376-95.

15. Basu AM. Health cultural influences care use: two regional groups in India. Stud Fam Plann. 1990;21(5):275-86.

16. Azami G, Soh KL, Sazlina SG, Salmiah MS, Aazami S, Mozafari M, et al. Developing and validating the educational materials for a nurse-led selfmanagement education in adults with type 2 diabetes. Int J Diabetes Metab. 2019;25(1-2):1-10.

17. Ahmad B, Ramadas A, Fatt QK, Zain AZM. A pilot study: The development of a culturally tailored Malaysian Diabetes Education Module (MYDEMO) based on the Health Belief Model. BMC Endocr Disord. 2014;14:31.

18. Park JS, Ahn CW. Educational program for diabetic patients in Korea-multidisplinary intensive management. Diabetes Res Clin Pract. 2007;77(1):194-8.

19. Craig P, Dieppe P, Macintyre S, Michie S, Nazareth I, Petticrew M. Developing and evaluating complex interventions. Med Res Counc. 2019.

20. University of Zurich. Fact sheet: How to conduct a focus group discussion (FGD): methodological manual, 2017. Available at: https://doi.org/10.5167 /uzh-150640. Accessed on 21 December 2020.

21. Sinisterra M, Kelly KP, Shneider C, El-Zein A, Swartwout E, Deyo P, et al. Working toward an mhealth platform for adolescents with type 1 diabetes: focus groups with teens, parents, and providers. Diabetes Educ. 2020;46(5):444-54.
22. Singh T, Sharma S, Nagesh S. Socio-economic status scales updated for 2017. Int J Res Med Sci. 2017;5(7):3264-7.

23. Doak C, Doak L, Root J. Teaching patients with low literacy skills. 2nd ed. Philadelphia: J. B. Lippincott; 1996.

24. Wolff K, Chambers L, Bumol S, White RO, Gregory BP, Davis D, et al. The PRIDE (partnership to improve diabetes education) toolkit: development and evaluation of novel literacy and culturally sensitive diabetes education materials. Diabetes Educ. 2016;42(1):23-33.

25. Connan V, Marcon MA, Mahmud FH, Assor E, Martincevic I, Bandsma RH, et al. Online education for gluten-free diet teaching: development and usability testing of an e-learning module for children with concurrent celiac disease and type 1 diabetes. Pediatr Diabetes. 2019;20(3):293-303.

26. Bowen DJ, Kreuter M, Spring B, Cofta-Woerpel L, Linnan L, Weiner D, et al. How we design feasibility studies. Am J Prev Med. 2009;36(5):452-7.

27. Brorsson AL, Leksell J, Franko MA, Olinder AL. A person-centered education for adolescents with type 1 diabetes-a randomized controlled trial. Pediatr Diabetes. 2019;20(7):986-96.

28. Gupta S, Sharma S, Gohil R, Sachdeva S. Epicollect5: a free, fully customizable mobile-based application for data collection in clinical research. J Postgrad Med Educ Res. 2021;54(4):248-51.

29. Varni JW, Delamater AM, Hood KK, Raymond JK, Chang NT, Driscoll KA, et al. PedsQL 3.2 diabetes module for children, adolescents, and young adults: Reliability and validity in type 1 diabetes. Diabetes Care. 2018;41(10):2064-71.

30. Barola A, Tiwari P, Bhansali A, Grover S, Dayal D. Cross-cultural adaptation and psychometric evaluation of Hindi version of diabetes selfmanagement profile-self report in Indian type 1 diabetes patients. Pediatr Diabetes. 2021;22(1):10111.

31. Awad LA, Elghadban FEE, El-Adham NA. Effect of an intervention program on improving knowledge and self-care practices for diabetic school-age children. Am J Nurs Res. 2019;7(2):199-207.

32. Magill N, Knight R, McCrone P, Ismail K, Landau S. A scoping review of the problems and solutions associated with contamination in trials of complex interventions in mental health. BMC Med Res Methodol. 2019;19(1):4.

Cite this article as: Rohilla L, Dayal D, Malhi P, Bharti B, Kaur S, Dhandapani M. Development and evaluation of a culturally tailored education module for type 1 diabetes in a resource-constrained setting: protocol for a mixed-methods study. Int J Clin Trials 2021;8(3):244-52. 